\title{
Analysis of the effects of clinical features, initial imaging findings and the number of days that nucleic acids turned negative: 201 cases of COVID- 19 patients
}

\section{Fen Xing}

Wuhan University Renmin Hospital

Jing Zhang

Wuhan University Renmin Hospital

\section{Bing Wang}

Wuhan University Renmin Hospital

Jingyun Cheng

Wuhan University Renmin Hospital

Baojun Xie ( $\nabla$ xiebj@126.com )

Renmin Hospital of Wuhan University https://orcid.org/0000-0001-9581-5322

\section{Research}

Keywords: COVID-19, Chest CT, Imaging findings, Nucleic acid, Clinical features

Posted Date: May 11th, 2020

DOI: https://doi.org/10.21203/rs.3.rs-26829/v1

License: (c) (1) This work is licensed under a Creative Commons Attribution 4.0 International License. Read Full License 


\section{Abstract}

Background: COVID-19 had spread quickly among the population and it has caused considerable morbidity and mortality in more than 200 countries worldwide, which became a public health emergency of international concern. This study was to investigate whether some clinical features, laboratory tests and imaging findings at the initial diagnosis of COVID-19 infection affect the number of days that nucleic acids turned negative $\left(D_{\text {negative }}\right)$ during hospitalization.

Methods: The Mann-Whitney U test and one-way analysis of variance (ANOVA) were used to examine the difference in $D_{\text {negative }}$ between clinical features, laboratory tests and imaging findings. ANOVA was also applied to differentiate between the age, $D_{\text {negative, }}$ and imaging findings among different clinical types. Multiple linear regression (MLR) analysis was used to quantify the effect of clinical characteristics, imaging findings and $D_{\text {negative, }}$ and establish regression equation.

Results: The $D_{\text {negative }}$ of COVID-19 patients who had comorbidity was significantly larger than that of those without $(33.35 \pm 14.63,27.79 \pm 12.89, P=0.007)$. Common patients were younger than severe and critically severe patients $(P<0.001, P=0.003)$. As compared to common patients, severe patients had larger $\mathrm{D}_{\text {negative }}(P<0.001)$, and the ratio of ground-glass opacity $\left(\mathrm{R}_{\mathrm{GGO}}\right)$, consolidation $\left(\mathrm{R}_{\mathrm{C}}\right)$, and the sum of GGO and consolidation ( $R_{\text {SUM }}$ ) of lesions on chest CT increased significantly (all $P<0.001$ ). The MLR analysis equation was $y\left(D_{\text {negative }}\right)=22.35+0.36 \times R_{\text {SUM }}$.

Conclusions: The severe COVID-19 patients were older than common patients, and their $\mathrm{D}_{\text {negative }}$ were larger as well. The chest $C T$ findings at initial diagnosis (including $R_{G G O}, R_{C}$, and $R_{S U M}$ ) could be used to differentially diagnose the common and severe patients, and had certain potential ability in predicting $D_{\text {negative. }}$

Trial registration: Retrospectively registered.

\section{Background}

Since December 2019, several cases of pneumonia of unknown aetiology were diagnosed with severe acute respiratory syndrome coronavirus-2 (SARS-CoV-2) in Wuhan, China. Recently, the World Health Organization formally named it as novel coronavirus disease 2019 (COVID-19)[1, 2]. The virus spread quickly among the population as soon as it was discovered. To date, it has caused considerable morbidity and mortality in more than 200 countries worldwide [3], which became a public health emergency of international concern. As of April 19,2020, more than 84,000 patients in China have been diagnosed with the virus, and the number of COVID-19 patients overseas has reached more than 2.27 million. However, the virus was still propagating at an alarming rate primarily through respiratory droplets and close contact. 
COVID-19 was an enveloped RNA virus with diverse clinical manifestations but lack of features, which brought certain difficulties in clinical diagnosis[4]. It was mainly confirmed by nucleic acid testing of respiratory secretions or excreta of the digestive tract. Previous study [5] reported that, out of 1014 patients infected with COVID-19, only $59 \%$ had positive nucleic acid test, whilst the sensitivity of chest CT was as high as $97 \%$. Therefore, laboratory test and imaging findings were often essential in clinic to assist diagnosis, which played an important role in early diagnosis of COVID-19 patients, especially those who were asymptomatic or had no obvious symptoms. At present, the etiology and pathogenesis of COVID-19 were still unclear, and there was no unified standard for the diagnosis and treatment and feasible method to evaluate the efficacy and prognosis of COVID-19 patients.

By retrospectively analyzing the nucleic acid results and imaging findings of 201 patients confirmed with COVID-19, we explored whether some clinical features, the initial laboratory tests and imaging findings would affect the number of days that nucleic acids turned negative ( $\left.D_{\text {negative }}\right)$ during hospitalization, so as to provide some guidance for the clinical evaluation of the patient's condition and hospitalization time.

\section{Methods}

\section{Patients}

We retrospectively collected 229 hospitalized patients in our hospital from January to March, 2020, and screened them according to the following inclusion criteria: (1) They were confirmed COVID-19 by nucleic acid test of nasopharyngeal swab; (2) The nucleic acid test results during hospitalization can be tracked, especially before hospital discharge; (3) The patient's medical record was relatively complete, and the clinical information of can be obtained; (4) The patient's chest CT image was clear enough to identify the lesion and for subsequent statistical analysis. Finally, a total of 201 patients were involved in this study. The clinical features, the laboratory tests and chest CT examinations of all patients were recorded. The clinical information mainly included the gender, age, clinical symptoms at the time of onset, exposure history, and comorbidity, etc. The laboratory tests involved the white blood cells, lymphocytes, C-reactive protein, and albumin, etc. For chest CT findings, the lung combined other lesions, lesion distribution, the ground-glass opacity (GGO), consolidation, the sum of GGO and consolidation in the lung were included. In addition, we tracked all patient's nucleic acid tests during treatment (including before and during hospitalization), and counted the number of days between the patient's diagnosis of COVID-19 infection and the nucleic acid turning negative. Herein, the date of nucleic acid turning negative meant the date when the first nucleic acid test that met the discharge criteria in the clinic was negative.

According to the fifth edition of the China Guidelines for the Diagnosis and Treatment Plan of Novel Coronavirus (2019-nCoV) Infection by the National Health Commission (Trial Version 5)[6], all COVID-19 patients were divided into four types: (1) mild type: with slight clinical symptoms, but no pneumonia manifestations in imaging; (2) common type: has fever, respiratory symptoms and imaging manifestations of pneumonia; (3) severe type: meeting any of the following: respiratory distress with RR $\geq 30$ times/min, oxygen saturation at rest $\leq 93 \%$, or $\mathrm{PaO}_{2} / \mathrm{FiO}_{2} \leq 300 \mathrm{mmHg}$; (4) critically severe type: 
with any of the following: respiratory failure needing mechanical ventilation, shock, or combination with other organ failure requiring ICU intensive care.

\section{Image acquisition}

All patients underwent routine standard chest CT scanning at an initial visit. The examination was mainly conducted at the Optima CT680 scanner (GE Medical Systems, Milwaukee, WI) with $210 \mathrm{~mA}$ and $120 \mathrm{kV}$ to obtain 5- and 1-mm axial images. The images of all patients were then input into the "New Coronavirus Pneumonia Intelligent Evaluation System V6.5" software for analysis. The ratio of the volume of GGO, consolidation, and the sum of them to the volume of the whole lung could be automatically calculated, which were represented as $R_{G G O}, R_{C}$, and $R_{S U M}$, respectively. These three parameters were used for subsequent statistical analysis.

\section{Data analysis}

In this study, the Kolmogorov-Smirnov test was used to detect the normality of the data, and the MannWhitney $\mathrm{U}$ test was used for non-normally distributed parameters (including gender, comorbidity, Creactive protein, albumin, and lung combining with other lesions). The difference in $D_{\text {negative }}$ between white blood cells and lymphocytes among different groups were analyzed by one-way analysis of variance (ANOVA). Similarly, the distinction of age, $D_{\text {negative }}$ and imaging findings (including $R_{G G O}, R_{C}$, and $\mathrm{R}_{\mathrm{SUM}}$ ) in different clinical types were also analyzed by using ANOVA. Taking gender, age, comorbidity, and lung combining with other lesions, $R_{G G O}, R_{C}$, and $R_{S U M}$ as independent variables, and $D_{\text {negative }}$ as the dependent variable, the multiple linear regression (MLR) analysis was used to explore the relation between chest $C T$ findings and $D_{\text {negative, }}$ and establish a regression equation. All data analysis was performed with the SPSS software (Version 22.0, IBM, Chicago, IL, USA), and the statistical difference was set at $\mathrm{P}<0.05$.

\section{Results}

A total of 201 hospitalized patients with COVID-19 were enrolled in this study, including 79 males and 122 females, with an average age of 56 years (range, 23-87 years). Most patients (75.5\%) had fever, and the rest had other clinical symptoms, such as cough, fatigue, sore throat, Headache, etc. Except for three patients who were infected by exposure to people from Wuhan, the remaining patients were residents of Wuhan. $29.9 \%$ of patients had one or more chronic diseases, mainly including hypertension, coronary heart disease, diabetes, asthma, hepatitis B, kidney disease and gout etc. Among these 201 patients, it included mild type in one (0.5\%), common in 134 (66.7\%), severe in 61(30.3\%), and critically severe in the rest five (2.5\%). Most patients at the initial diagnosis showed normal white blood cells $(70.1 \%)$, normal $(50.3 \%)$ or decreased $(32.3 \%)$ lymphocytes, normal $(40.8 \%)$ or increased $(41.3 \%)$ C-reactive protein, normal (22.4\%) or decreased (59.7\%) albumin. On chest CT imaging, 178 patients showed bilateral pneumonia, whilst 22 patients had unilateral pneumonia. Each lobe was involved in the lesion for COVID- 
19 patients. The chest CT manifestations of four types were showed in Fig. 1, and the clinical information, laboratory tests and chest CT findings of all patients were summarized in Table 1.

The histogram distribution of $D_{\text {negative }}$ in all COVID-19 patients was showed in Fig. 2. As revealed in Table 2 , there was no significant difference in $D_{\text {negative }}$ between different groups of gender and lung combination with other lesions $(P=0.604$ and $P=0.993)$, but the $D_{\text {negative }}$ for patients with comorbidity was significantly higher than that of those without (the mean \pm standard deviation was $33.35 \pm 14.63$, $27.79 \pm 12.89, P=0.007)$. Some laboratory indicators, mainly including leukocytes, lymphocytes, $C$ reactive protein and albumin, which were normal, increased or decreased, were not statistically significant for $\mathrm{D}_{\text {negative }}($ all $P>0.05)$.

Common patients were significantly younger than severe and critically severe patients (common vs. severe vs. critically severe: $52.16 \pm 14.19$ vs. $62.84 \pm 13.45$ vs. $71.00 \pm 12.51, P$ was $<0.001,0.003$, and 0.210 , respectively). Compared with common patients, the $D_{\text {negative }}$ of severe patients was larger (common vs. severe: $25.53 \pm 11.33$ vs. $37.13 \pm 14.35, P<0.001$ ), and the $\mathrm{R}_{\mathrm{GGO}}, \mathrm{R}_{\mathrm{C}}$, and $\mathrm{R}_{\mathrm{SUM}}$ on chest CT imaging were also significantly increased (common vs. severe: $6.31 \pm 8.95$ vs. $19.49 \pm 15.26,1.60 \pm 3.03$ vs. $5.78 \pm 6.28,7.91 \pm 11.35$ vs. $25.27 \pm 20.10$, all $P<0.001)$. Nevertheless, although the $D_{\text {negative, }}, R_{G G O}$, $\mathrm{R}_{\mathrm{C}}$, and $\mathrm{R}_{\mathrm{SUM}}$ for critically severe patients were higher than that of common and severe patients, they were not statistically significant (all $P>0.05$ ) (see Table $\mathbf{3}$ and Fig. 3).

Taking clinical characteristics and CT imaging findings as independent variables, the results of MLR analysis of $D_{\text {negative }}$ showed that the $R$ value of final model was 0.331 , and the adjusted $R^{2}$ value was 0.110 . The detection value of Durbin-Watson was 2.14, indicating that the equation had a medium intensity fitting. As can be seen in Table 4, the variables of $R_{S U M}$ and the constant were finally included in the model equation as: $y\left(D_{\text {negative }}\right)=22.35+0.36 \times R_{\text {SUM }}$.

\section{Discussion}

Coronavirus disease 2019 have spread throughout the world execrably. As of April 19, 2020, the statistical data revealed that the mortality rate of COVID-19 infection in China was 5.5\%, while for overseas it was about $6.9 \%$, which was much lower than SARS (9.6\%) and MERS (37.1\%) [4]. However, the transmissibility of COVID-19 virus was much higher than SARS and MERS, and its incubation period was also long, causing a large number of asymptomatic infections. This could make huge challenges for the diagnosis and control of the disease, posing a great threat to the safety of human life worldwide.

Herein, this study revealed that common patients were much younger $(P<0.001$ and 0.003$)$, and the $D_{\text {negative }}$ of common patients was smaller than that of severe and critically severe patients. The clinical symptoms of common patients were relatively mild, and after receiving corresponding treatment, the virus in the body could be quickly removed. But for severe and critically severe patients, they were more 
seriously ill at an initial visit and suffered from impaired respiratory status. Thus their prognosis would be worse[1].

The study of Liu et al.[7] confirmed that the age of COVID-19 infected patients whose disease progressed during hospitalization was significantly higher than that of patients with disease improvement/stabilization. Due to the older age and reduced body immunity, severe and critically severe patients were more vulnerable to virus attacks. Although the $D_{\text {negative }}$ of critically severe patients higher than that of common and severe patients, they were not statistically different. This was mainly due to the small sample size of the critically severe patients (only 5 cases), which was greatly lower than the other two types.

Moreover, the chest CT examination played a key role in the diagnosis of COVID-19 infection, and its sensitivity was as high as $97 \%-98 \%[5,8]$. Many previous studies[9-12] reported the chest CT imaging features of COVID-19 patients, but most of them only described the lesion morphologically, such as the location, shape, range, and density etc. of the lesions, and lacked to quantify the lesions. However, this study was aimed to classify and quantify the lesions, and confirmed that the $R_{G G O}, R_{C}$, and $R_{S U M}$ of the lesions of common patients at an initial visit were significantly lower than that of severe patients. When the virus invaded the body, it would produce a series of inflammatory reactions, which can be seen on chest CT as exudation, consolidation, and even fibrosis. The more lesions on the lungs, the greater the damage to the lungs. The lung blood exchange was more restricted, which gave rise to more severe clinical manifestations. As a result, the chest $C T$ of severe patients showed higher $R_{G G O}, R_{C}$, and $R_{S U M}$. However, these ratios did not reach statistical significance between common, severe and critically severe patients, mainly because of too small sample size of critically severe patients. Therefore, it can be reasonably inferred from this study that these ratios had potential value in the differential diagnosis of common and severe patients that infected with COVID-19.

The laboratory tests of COVID-19 patients mainly showed normal white blood cells, normal or decreased lymphocytes, increased or normal C-reactive protein, normal or decreased albumin, which was consistent with previous results $[1,13,14]$. These laboratory results differ from those of a common bacterial infection in the lung, particularly lymphocytes, which may be related to the pathogenesis of the virus. We believed that the laboratory indicators at an initial visit could only be used to assist in the diagnosis of COVID-19 infection, but it couldn't be used to determine $D_{\text {negative }}$.

It was worth noting that $29.9 \%$ of the patients in this study had one or more comorbidities, which was less than half of the patients in MERS-CoV [15]. This result also showed that the $D_{\text {negative }}$ of patients with comorbidity was significantly higher than that of those without $(P=0.006)$. The comorbidity in this study mainly included cardiovascular and cerebrovascular diseases, diabetes, and kidney disease, which was similar to those previous reports $[7,15]$. The cause of these comorbidities may be related to the pathogenesis of COVID-19『which may downregulate the key mediators of host's innate immune response to the pathogenesis. These comorbidities shared some common features with infectious diseases and their complications, such as endothelial dysfunction, proinflammatory factor status, and weakened 
innate immune response[16, 17]. As a result, it may lead to prolonged virus clearance in the body. We believed that the comorbidities may not be a susceptible factor for patients infected with the COVID-19 virus, but it would prolong the $D_{\text {negative }}$ of patients, so it was necessary to long-term observe the nucleic acid change of patients with comorbidities clinically.

The chest CT examination played an important role in diagnosing COVID-19 infection and evaluating the therapeutic effect of patients. It was a current trend to use big data analytics to discern and quantify the lesions of patients with COVID-19 infection, which could not only further improve the accuracy of radiologists in the diagnosis of pulmonary lesions, but also observe subtle changes in lesions that were difficult to be recognized by the naked eye during the follow-up examination. By performing the MILR analysis of the ratio of lesions on the initial chest $C T$ and $D_{\text {negative, }}$ an effective correlation equation was obtained as $y\left(D_{\text {negative }}\right)=22.35+0.36 \times R_{\text {SUM }}$. This equation suggested that the ratio of lesions on chest $\mathrm{CT}$ at the first visit had some potential prediction on patients' $\mathrm{D}_{\text {negative. }}$. Although there have been scarce data to support the finding to date, continued observations and further assessment are needed, and we believe that it could provide some reference for clinical practice.

This study also had some limitations. Firstly, the sample of mild and critically severe COVID-19 patients was too small to analyze, which couldn't reflect the real clinical features and imaging findings. Secondly, due to the shortage of early medical resources, many patients didn't go to the doctor timely, which resulted in the inconsistency of the time from the onset to the diagnosis and the chest CT examination.

\section{Conclusions}

In this study, there was no significant difference in $D_{\text {negative }}$ between different groups of gender and lung combination with other lesions $(P=0.604$ and 0.993$)$, but the $D_{\text {negative }}$ for patients with comorbidity was significantly higher than that of those without $(P=0.007)$. Some laboratory indicators, mainly including leukocytes, lymphocytes, $C$-reactive protein and albumin, were not statistically significant for $D_{\text {negative }}$ (all $P>0.05)$. As compared to common patients, severe patients were older, and they had larger $\mathrm{D}_{\text {negative }}(P$ $<0.001$ ), and the $\mathrm{R}_{\mathrm{GGO}}, \mathrm{R}_{\mathrm{C}}$, and $\mathrm{R}_{\mathrm{SUM}}$ of lesions on chest $\mathrm{CT}$ increased significantly (all $P<0.001$ ). The MLR analysis equation was y $\left(D_{\text {negative }}\right)=22.35+0.36 \times R_{\text {SUM }}$. All these may suggest that the chest CT findings at initial diagnosis could be used to differentially diagnose the common and severe patients, and had certain potential ability in predicting $D_{\text {negative. }}$

\section{Abbreviations}

COVID-19: Coronavirus disease 2019

$D_{\text {negative: }}$ the number of days that nucleic acids turned negative

GGO: the ground-glass opacity 
$\mathrm{R}_{\mathrm{GGO}}$ : The ratio of the volume of $\mathrm{GGO}$ to the volume of the whole lung

$R_{C}$ : The ratio of the volume of consolidation to the volume of the whole lung

$\mathrm{R}_{\text {SUM }}$ : The ratio of the sum volume of GGO and consolidation to the volume of the whole lung

ANOVA: One-way analysis of variance

MLR: The multiple linear regression analysis

\section{Declarations}

Ethics approval and consent to participate

This study was approved by the institutional review board of the designated hospital, and written informed consent was waived for the retrospective study.

Consent for publication

Not applicable.

\section{Availability of data and materials}

All data generated or analyzed during this study are included in this published article. The detailed datasets are available from the corresponding author on reasonable request.

Competing interests

The authors declare that they have no competing interests.

\section{Funding}

No funding was provided for this study.

\section{Authors' contributions}

Fen Xing collected, analyzed data and wrote the manuscript. Jing Zhang collected some data, reviewed and revised the manuscript. Bing Wang collected some data, reviewed and revised the manuscript. Jingyun Cheng collected some data, reviewed and revised the manuscript. Baojun Xie designed the study, and revised the manuscript.

Acknowledgements

Not applicable. 


\section{References}

1. Guan W-j, Ni Z-y, Hu Y, Liang W-h, Ou C-q, He J-x, et al. Clinical Characteristics of Coronavirus Disease 2019 in China. New England Journal of Medicine. 2020;

2. Huang C, Wang Y, Li X, Ren L, Zhao J, Hu Y, et al. Clinical features of patients infected with 2019 novel coronavirus in Wuhan, China. The Lancet. 2020; 395: 497-506

3. Shi S, Qin M, Shen B, Cai Y, Liu T, Yang F, et al. Association of Cardiac Injury With Mortality in Hospitalized Patients With COVID-19 in Wuhan, China. JAMA Cardiol. 2020;

4. Fang Z, Yi F, Wu K, Lai K, Sun X, Zhong N, et al. Clinical Characteristics of 2019 Coronavirus Pneumonia. 2020;

5. Ai T, Yang Z, Hou H, Zhan C, Chen C, Lv W, et al. Correlation of Chest CT and RT-PCR Testing in Coronavirus Disease 2019 (COVID-19) in China: A Report of 1014 Cases. Radiology. 2020; 200642

6. Lin L, Li TS. [Interpretation of "Guidelines for the Diagnosis and Treatment of Novel Coronavirus (2019-nCoV) Infection by the National Health Commission (Trial Version 5)"]. Zhonghua yi xue za zhi. 2020; 100: E001

7. Liu W, Tao ZW, Lei W, Ming-Li Y, Kui L, Ling Z, et al. Analysis of factors associated with disease outcomes in hospitalized patients with 2019 novel coronavirus disease. Chin Med J (Engl). 2020;

8. Fang Y, Zhang H, Xie J, Lin M, Ying L, Pang P, et al. Sensitivity of Chest CT for COVID-19: Comparison to RT-PCR. Radiology. 2020; 200432

9. Bernheim A, Mei X, Huang M, Yang Y, Fayad ZA, Zhang N, et al. Chest CT Findings in Coronavirus Disease-19 (COVID-19): Relationship to Duration of Infection. Radiology. 2020; 200463

10. Wang Y, Dong C, Hu Y, Li C, Ren Q, Zhang X, et al. Temporal Changes of CT Findings in 90 Patients with COVID-19 Pneumonia: A Longitudinal Study. Radiology. 2020; 200843

11. Lin C, Ding Y, Xie B, Sun Z, Li X, Chen Z, et al. Asymptomatic novel coronavirus pneumonia patient outside Wuhan: The value of CT images in the course of the disease. Clin Imaging. 2020; 63: 7-9

12. Xu YH, Dong JH, An WM, Lv XY, Yin XP, Zhang JZ, et al. Clinical and computed tomographic imaging features of novel coronavirus pneumonia caused by SARS-CoV-2. J Infect. 2020; 80: 394-400

13. Chen N, Zhou M, Dong X, Qu J, Gong F, Han Y, et al. Epidemiological and clinical characteristics of 99 cases of 2019 novel coronavirus pneumonia in Wuhan, China: a descriptive study. The Lancet. 2020; 395: 507-13

14. Liu K, Fang YY, Deng Y, Liu W, Wang MF, Ma JP, et al. Clinical characteristics of novel coronavirus cases in tertiary hospitals in Hubei Province. Chin Med J (Engl). 2020;

15. Badawi A, Ryoo SG. Prevalence of comorbidities in the Middle East respiratory syndrome coronavirus (MERS-CoV): a systematic review and meta-analysis. Int J Infect Dis. 2016; 49: 129-33

16. Htun NS, Odermatt P, Eze IC, Boillat-Blanco N, D'Acremont V, Probst-Hensch N. Is diabetes a risk factor for a severe clinical presentation of dengue?-review and meta-analysis. PLoS Neg/ Trop Dis. 2015; 9: $\mathrm{e} 0003741$ 
17. Dharmashankar K, Widlansky ME. Vascular endothelial function and hypertension: insights and directions. Curr Hypertens Rep. 2010; 12: 448-55

\section{Tables}


Table 1 All patients' clinical information, laboratory tests, and imaging findings

Patients (201

cases)

Clinical

information
Demographic information

.

\begin{tabular}{ll}
\hline Clinical symptoms & Fever \\
\hline Cough \\
\hline
\end{tabular}

Fatigue

Sore throat

\begin{tabular}{ll}
\hline Headache \\
Diarrhoea \\
\hline
\end{tabular}

Ches

Exposure history

Comorbidity

\begin{tabular}{ll} 
& hospital infection \\
\hline Comorbidity & No \\
\cline { 2 - 2 } & Yes
\end{tabular}

Clinical classification
56 (23-87)

$79(39.3 \%)$

$122(60.7 \%)$

$152(75.5 \%)$

$18(9.0 \%)$

$11(5.5 \%)$

$4(2.0 \%)$

$3(1.5 \%)$

$2(1.0 \%)$

$2(1.0 \%)$

$1(0.5 \%)$

$8(4.0 \%)$

$197(98.0 \%)$

$3(1.5 \%)$

$1(0.5 \%)$

141 (70.1\%)

$60(29.9 \%)$

$1(0.5 \%)$

$134(66.7 \%)$

$61(30.3 \%)$

$5(2.5 \%)$

Critically severe type

$141(70.1 \%)$

19 (9.5\%)

$10(5.0 \%)$

31 (15.4\%)

$101(50.3 \%)$ 


\begin{tabular}{|c|c|c|c|}
\hline & & Decreased & 65 (32.3\%) \\
\hline & & Increased & $4(2.0 \%)$ \\
\hline & & Unclear & $31(15.4 \%)$ \\
\hline & \multirow[t]{4}{*}{ C-reactive protein } & Normal & $82(40.8 \%)$ \\
\hline & & Decreased & 0 \\
\hline & & Increased & $83(41.3 \%)$ \\
\hline & & Unclear & $36(17.9 \%)$ \\
\hline & \multirow[t]{4}{*}{ Albumin } & Normal & $45(22.4 \%)$ \\
\hline & & Decreased & $120(59.7 \%)$ \\
\hline & & Increased & 0 \\
\hline & & Unclear & $36(17.9 \%)$ \\
\hline \multirow[t]{11}{*}{ Imaging findings } & \multirow{2}{*}{$\begin{array}{l}\text { Lung combined other } \\
\text { lesions }\end{array}$} & No & $186(92.5 \%)$ \\
\hline & & Yes & $15(7.5 \%)$ \\
\hline & \multirow[t]{4}{*}{ Lesions distribution } & Both lungs & $172(85.6 \%)$ \\
\hline & & Left lung & $11(5.4 \%)$ \\
\hline & & Right lung & $17(8.5 \%)$ \\
\hline & & None & $1(0.5 \%)$ \\
\hline & \multirow[t]{5}{*}{ Pulmonary lobe } & Left upper lobe & 152 \\
\hline & & Left lower lobe & 173 \\
\hline & & Right upper lobe & 149 \\
\hline & & Right middle lobe & 137 \\
\hline & & Right lower lobe & 176 \\
\hline
\end{tabular}


Table 2 The difference of $D_{\text {negative }}$ between different groups of clinical information, laboratory tests, and imaging findings

\begin{tabular}{|c|c|c|c|c|}
\hline & & & $\begin{array}{l}\text { Mean } \pm \text { standard } \\
\text { deviation }\end{array}$ & $\begin{array}{l}P \\
\text { value }\end{array}$ \\
\hline \multirow{4}{*}{$\begin{array}{l}\text { Clinical } \\
\text { information }\end{array}$} & Gender & Male & $28.94 \pm 13.81$ & \multirow[t]{2}{*}{$0.604^{\mathrm{a}}$} \\
\hline & & Female & $29.79 \pm 13.57$ & \\
\hline & \multirow[t]{2}{*}{ Comorbidity } & No & $27.79 \pm 12.89$ & \multirow[t]{2}{*}{$0.007^{\circ}$} \\
\hline & & Yes & $33.35 \pm 14.63$ & \\
\hline \multirow[t]{10}{*}{ Laboratory test } & \multirow[t]{3}{*}{ White blood cell } & Normal & $30.92 \pm 13.41$ & \multirow[t]{3}{*}{$0.615^{b}$} \\
\hline & & Decreased & $33.95 \pm 13.44$ & \\
\hline & & Increased & $29.50 \pm 17.89$ & \\
\hline & \multirow[t]{3}{*}{ Lymphocyte } & Normal & $30.52 \pm 12.19$ & \multirow[t]{3}{*}{$0.818^{\mathrm{b}}$} \\
\hline & & Decreased & $32.03 \pm 15.65$ & \\
\hline & & Increased & $33.75 \pm 16.92$ & \\
\hline & \multirow[t]{2}{*}{ C-reactive protein } & Normal & $30.15 \pm 11.44$ & \multirow[t]{2}{*}{$0.236^{a}$} \\
\hline & & Increased & $33.31 \pm 15.07$ & \\
\hline & \multirow[t]{2}{*}{ Albumin } & Normal & $29.18 \pm 11.90$ & \multirow[t]{2}{*}{$0.287^{a}$} \\
\hline & & Decreased & $32.45 \pm 14.17$ & \\
\hline \multirow[t]{2}{*}{ Imaging findings } & \multirow{2}{*}{$\begin{array}{l}\text { Lung combined other } \\
\text { lesions }\end{array}$} & No & $29.45 \pm 13.63$ & \multirow[t]{2}{*}{$0.993^{\circ}$} \\
\hline & & Yes & $29.53 \pm 14.18$ & \\
\hline
\end{tabular}

$D_{\text {negative }}$ indicated the number of days that nucleic acids turned negative. ${ }^{a}$ indicated the test of MannWhitney $U \mathbb{Z}^{\mathrm{b}}$ indicated the test of Kruskal-Wallis. 
Table 3 The ANOVA analysis of age, $D_{\text {negative, }}$ and imaging findings among different clinical types

\begin{tabular}{lllllll}
$\begin{array}{l}\text { Clinical } \\
\text { types }\end{array}$ & Common & Severe & $\begin{array}{l}\text { Critically } \\
\text { severe }\end{array}$ & $\begin{array}{l}P 1 \\
\text { (Common }\end{array}$ & $\begin{array}{l}\text { P2 } \\
\text { (Common } \\
\text { vs. Critically } \\
\text { severe) }\end{array}$ & $\begin{array}{l}\text { P3 } \\
\text { (Severe vs } \\
\text { Critically } \\
\text { severe) }\end{array}$ \\
\hline Age(year) & $52.16 \pm 14.19$ & $62.84 \pm 13.45$ & $71.00 \pm 12.51$ & $<0.001$ & 0.003 & 0.209 \\
\hline $\begin{array}{l}D_{\text {negative }} \\
\text { (days) }\end{array}$ & $25.53 \pm 11.33$ & $37.13 \pm 14.35$ & $41.00 \pm 18.95$ & $<0.001$ & 0.322 & 0.955 \\
\hline $\mathrm{R}_{\mathrm{GGO}}(\%)$ & $6.31 \pm 8.95$ & $19.49 \pm 15.26$ & $27.07 \pm 27.28$ & $<0.001$ & 0.365 & 0.898 \\
\hline $\mathrm{R}_{\mathrm{C}}(\%)$ & $1.60 \pm 3.03$ & $5.78 \pm 6.28$ & $5.38 \pm 5.17$ & $<0.001$ & 0.393 & 0.998 \\
\hline $\mathrm{R}_{\mathrm{SUM}}(\%)$ & $7.91 \pm 11.35$ & $25.27 \pm 20.10$ & $32.46 \pm 32.18$ & $<0.001$ & 0.363 & 0.942
\end{tabular}

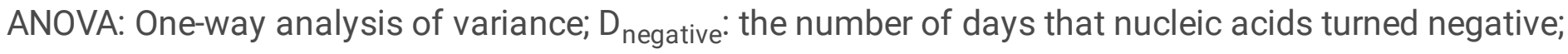
$R_{G G O} R_{C}$, and $R_{S U M}$ were respectively indicated the ratio of ground-glass opacity (GGO), consolidation, and the sum of GGO and consolidation of the lesions on chest CT.

Table 4 The Multiple linear regression analysis of clinical features, imaging findings and $D_{\text {negative }}$

\begin{tabular}{llllll} 
Model & Coefficient & $\begin{array}{l}\text { Standard } \\
\text { error }\end{array}$ & $\begin{array}{l}\text { Standard } \\
\text { coefficient }\end{array}$ & $\begin{array}{l}P \\
\text { value }\end{array}$ & $\begin{array}{l}\text { Collinearity } \\
\text { statistics (VIF) }\end{array}$ \\
\hline (constant) & 22.35 & 3.87 & & $<0.001$ & \\
\hline Gender & 2.62 & 1.89 & 0.1 & 0.168 & 1.05 \\
\hline Age & 0.02 & 0.07 & 0.03 & 0.729 & 1.38 \\
\hline Comorbidity & 2.33 & 2.22 & 0.08 & 0.296 & 1.27 \\
\hline $\begin{array}{l}\text { Lung combining with } \\
\text { other lesions }\end{array}$ & -3.23 & 3.69 & -0.06 & 0.383 & 1.11 \\
\hline$R_{C}$ & -0.52 & 0.38 & -0.18 & 0.173 & 3.99 \\
\hline$R_{\text {SUM }}$ & 0.36 & 0.11 & 0.47 & $<0.001$ & 4.33
\end{tabular}

Dependent variable: $D_{\text {negative }}$ (the number of days that nucleic acids turned negative); $R_{C}$, and $R_{S U M}$ were respectively indicated the ratio of consolidation, and the sum of ground-glass opacity (GGO) and consolidation of the lesions on chest CT. 


\section{Figures}

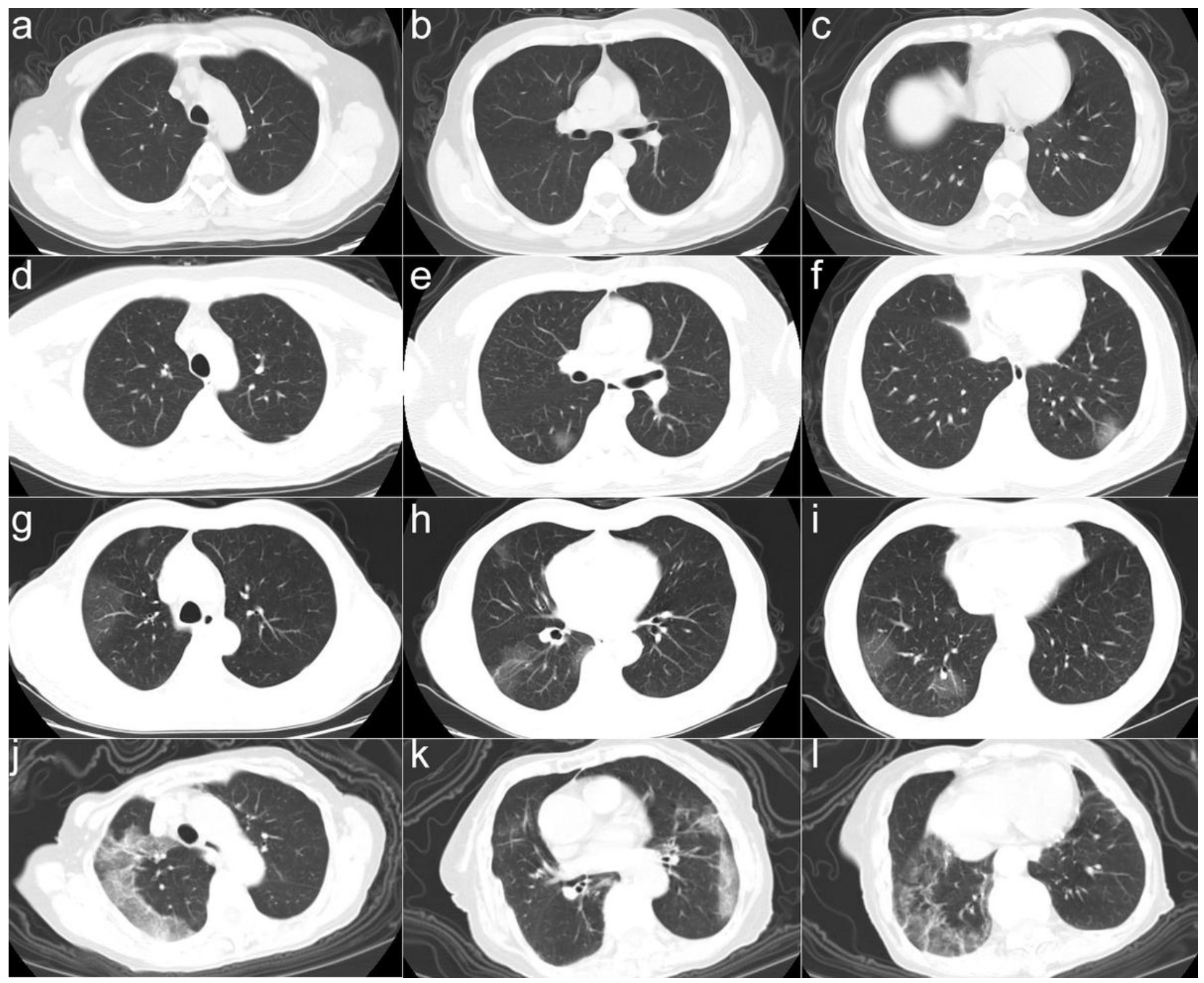

Figure 1

a-c: 57-year male patients, who was diagnosed mild type, and with no obvious imaging manifestations. $d-$ f: 31-year male patients, who was diagnosed common type. There was few patchy ground-glass opacity in the lower lobes of both lungs on chest CT. g-i: 69-year male patients, who was diagnosed severe type. There was multiple patchy ground-glass opacity in the all lobes of right lung. J-l: 80-year female patients, who was diagnosed critically severe type. There was diffuse patchy ground ground-glass opacity and few consolidation in all lobes of both lungs. 


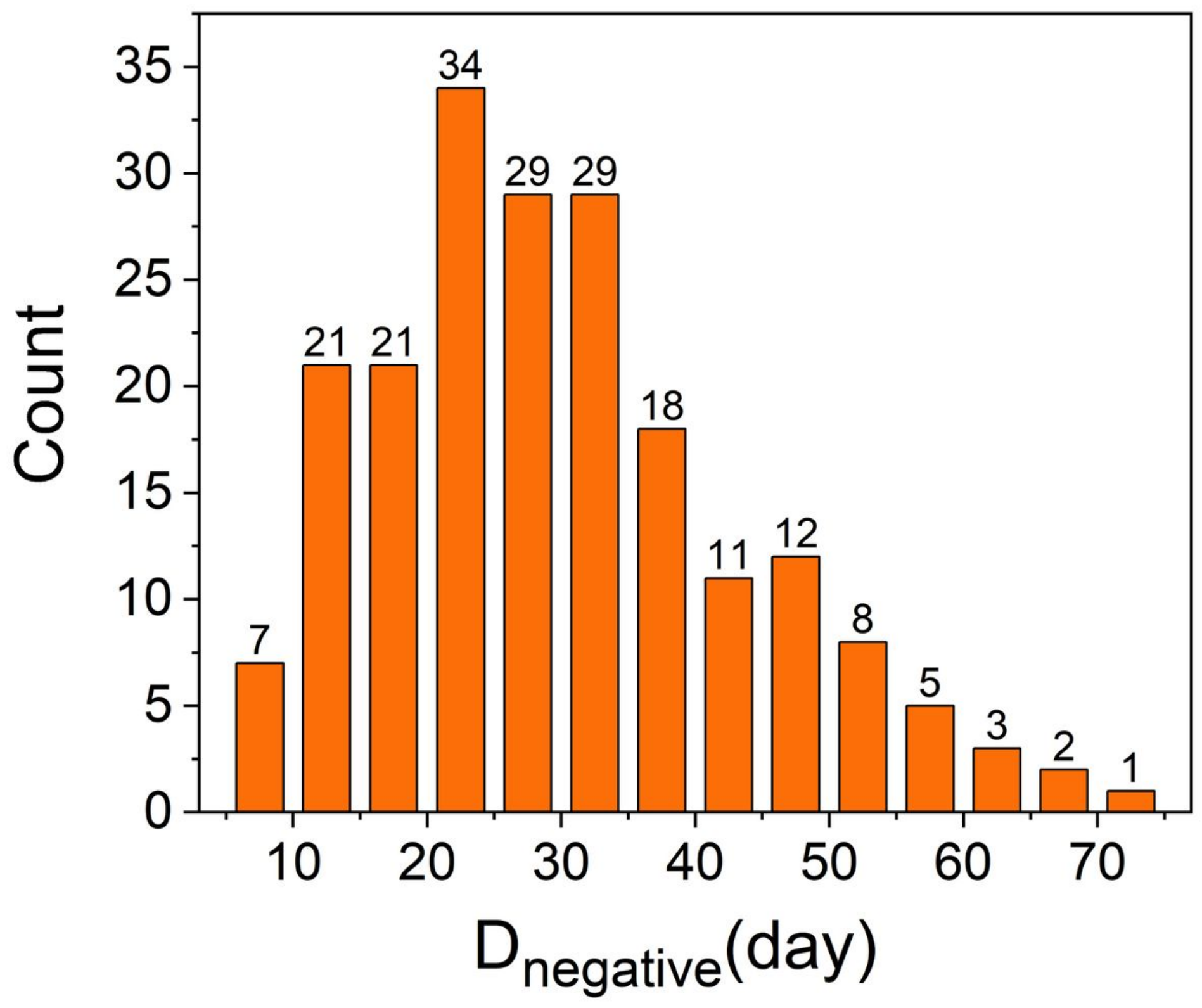

Figure 2

The histogram of the number of days that nucleic acids turned negative (Dnegative). 

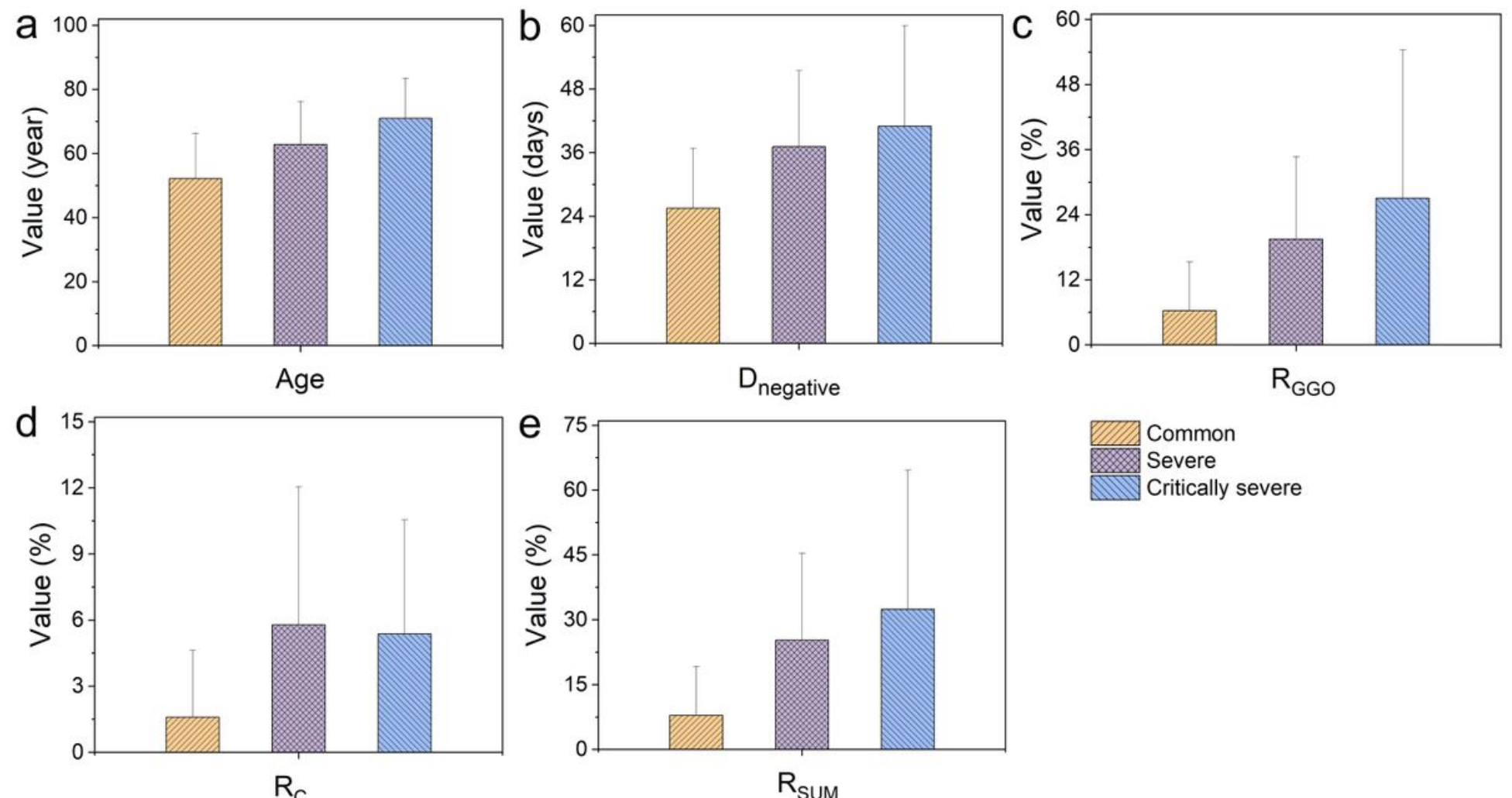

Common

Severe

Critically severe

$\mathrm{R}_{\mathrm{C}}$

$\mathrm{R}_{\text {SUM }}$

Figure 3

The bar chart of age, Dnegative, and imaging findings among different clinical types. Severe patients were older than common patients (a), and had larger Dnegative (b). On chest CT, the RGGO (c), Rc (d), and Rsum(e) of severe patients were significantly higher than common patients. Dnegative: the number of days that nucleic acids turned negative; RGGO, RC, and RSUM were respectively indicated the ratio of ground-glass opacity (GGO), consolidation, and the sum of GGO and consolidation of the lesions on chest CT. 